\title{
Early Term Birth
}

National Cancer Institute

\section{Source}

National Cancer Institute. Early Term Birth. NCI Thesaurus. Code C114094.

Birth at 37 weeks and 0 days through 38 weeks and 6 days. 